\title{
Assessing Students' Reading Comprehension Through Rubrics
}

\author{
Marija Mijušković, MA \\ University of Montenegro, Faculty of Philosophy - Nikšić \\ Email:ape.maja@t-com.me
}

Doi:10.5901/mjss.2014.v5n13p252

\begin{abstract}
Rubrics can be used for assessing students reading comprehension in many ways. Firstly, they help to ensure that the learning targets become clear. The students must be able to understand the learning target in order to develop superior outcomes. Students who have been given criteria for reading comprehension will be able to complete complex tasks. They will be able to demonstrate the required skills which can be assessed by the teachers. Another critical aspect of rubrics in reading comprehension is the fact that they help to facilitate instructional design and delivery. Instructional design is an important part of the educational process because it must be able to use personalized as well as universal strategies to meet the needs of students. Teachers that eloquently craft the expectations to students can help the latter to focus on key learning targets. Instructional approaches can then be used to improve the efficacy of the learning environment. The assessment process should be accurate and fair when designing rubrics. This is important specifically with reference to the needs of assessing students reading comprehension. Teachers can achieve consistency when they refer to common rubrics. The reviewer is able to judge in a fair manner by referring to the key aspects of the rubrics. This is an important strategy that must lead to long term outcomes within a short period of time. Students are able to identify the assessment criteria that are an important part of completing task.
\end{abstract}

Keywords: assessment, rubrics, reading comprehension, instructional design, learning targets, strategies

\section{Introduction}

Rubric is an academic term which refers to performance standards that are designed for specific students. Rubrics have been extensively applied in the academic setting as a means of improving learning outcomes among students. Additionally, the goals have been to create a collaborative strategy that helps to improve the efficacy of the learning environment. Rubrics tend to focus on supporting student self-awareness and self-reflection so that they can become acquainted with the basic objectives of the course. Moreover, rubrics can be used to create standards that can be aligned with the learning objectives.

Students reading comprehension can be assessed by the use of rubrics provided there are clear and precise goals. Furthermore, there needs to be a collaborative approach in which the emphasis is towards achieving long term educational goals. Rubrics must be designed with performance goals, descriptions, and outcomes. Clear and precise rubrics help in accomplishing learning efficiency while enhancing the learning environment. The use of rubrics is highly popular in the educational environment. They have become valuable tools in order to ensure proper assessment in an efficient manner.

\section{Background}

Rubrics are important because they can be used to assist students by providing a comprehensive evaluation framework. It can significantly reduce the time that teachers need in order to assess student work (Eisner, 2009). Rubrics are created by using various models that compare the good work with the poor work. This is done through provision of assignments that use quality samples in order to achieve efficiency and effectiveness. It is through the use of an innovative and creative approach that success can be attained within a short period of time. A criteria needs to be devised for the scoring rubric so that discussions can be done in order to produce quality work.

Student feedback is critically obtained so that teachers can evaluate the writing experiences of students. It is through the use of an innovative and creative strategy that success can be attained within a short period of time (Heath, 2009). Hierarchical categories can be created in order to describe the multiple quality levels. Additionally, quality can be measured through development criteria that employ innovative strategies in order to achieve long term success within a short period of time. Rubrics must be used by using developed samples as this can be beneficial for the educational objectives. Another way to increase the efficacy of rubrics is to use models so that students can test using the sample assignments. This helps to gradually enhance the confidence levels of students. A student and teacher agreement can be 
reached which helps to ensure the highest levels of reliability. Rubrics help to codify the criteria and standards that are needed for academic assignments. Moreover, they play an important role in conveying the educational expectations that a specific assignment project desires (Heath, 2009).

This helps to improve the ability of teachers to assign specific assignments in an efficient and effective manner. Rubrics also aid in the process of creating self-review and peer-review. Thus rubrics are an integral element of the entire process as they focus on development and growth. The development of a comprehensive strategy is critical for success as it leads to long term outcomes. Rubrics can be used to assess the student's learning strategy as well as develop an efficient lesson plan. The quality of the work can be judged through the use of an innovative and creative approach (Heath, 2009).

The student can explain his or her understanding of the material to the teacher if there are gaps between the work and teacher's evaluation. Rubrics need to be continuously developed as a means of achieving long term success. The development of a comprehensive strategy is important in order to ensure successful outcomes. Rubrics are becoming an integral element of the entire process because of their advantages to both teachers and students.

\subsection{Benefits}

Rubrics are widely used to rate performance in the learning environment. Definitions and examples can be employed together with the rubrics in order to provide students with the ability to understand the attributes which will be evaluated. A rating scale is employed for the various parts of the rubric. Rubrics are several dimensions that are used to evaluate the student response for any project (Hafner \& Hafner, 2004). Definitions and examples must be used in order to convey the meaning of the specific trait. Scale of values must exist in order to providing rating to each dimension.

Rubrics can be used for assessing students reading comprehension in many ways. Firstly, they help to ensure that the learning targets become clear. The students must be able to understand the learning target in order to develop superior outcomes (Hafner \& Hafner, 2004). Students who have been given criteria for reading comprehension will be able to complete complex tasks. They will be to demonstrate the required skills which can be assessed by the teachers.

Another critical aspect of rubrics in reading comprehension is the fact that they help to facilitate instructional design and delivery. Instructional design is an important part of the educational process because it must be able to use personalized as well as universal strategies to meet the needs of students. Teachers that eloquently craft the expectations to students can help the latter to focus on key learning targets. Instructional approaches can then be used to improve the efficacy of the learning environment (Hafner \& Hafner, 2004).

The assessment process should be accurate and fair when designing rubrics. This is important specifically with reference to the needs of assessing students reading comprehension. Teachers can achieve consistency when they refer to common rubrics. The reviewer is able to judge in a fair manner by referring to the key aspects of the rubrics. This is an important strategy that must lead to long term outcomes within a short period of time. Students are able to identify the assessment criteria that are an important part of completing task (Hafner \& Hafner, 2004). Furthermore, they can use the rubric in order to perform self-evaluation of their work. Students develop the ability to accurately evaluate their own work in an impartial manner. This helps to develop their competencies in an efficient and effective manner. It helps to create a collaborative strategy for success. Rubrics can play an important role in ensuring that students from diversified backgrounds can make the progression in the learning environment. Learning expectations become explicit which helps to attain long term success. Student performance and behavior can be enhanced through an efficient approach (Stiggins, 2010). Additionally, expectations can be outlined in a systematic and gradual manner that benefits in the long term. Students belonging to diversified settings are able to understand the language when they become acquainted with rubrics. This is a major advantage that can help to ensure long term success within a short period of time. It also helps to develop a collaborative framework for success.

\subsection{Limitations}

Rubrics that are well-designed can help teachers to assess the students' reading comprehension skills. This is dependent upon the fact that rubrics must be valid and reliable. Additionally, the teaching and learning process can be enhanced through the use of rubrics. But the presence of rubrics is not sufficient enough to ensure sound outcomes. There are limitations to the use of rubrics in the assessment process (Jackson, 2009). This is because the rubrics must help the teachers to apply individual knowledge that can be applied in the rating process. The goal should be to prevent the rating process from falling victim to personality variations and limitations that are associated with human information processing. Rubrics take lengthy periods of time to develop because performance outcomes, levels, and descriptions need to be 
designed. They are effective for significant and difficult assignments which can justify the amount of time needed to prepare them (Stiggins, 2010).

Having a clear and precise methodology is the key to success in rubric development. The use of innovation can be used as a tool for sound outcomes (Jackson, 2009). It is through the use of innovation and creativity that long term success can be attained within a short period of time. Poorly designed rubrics can hinder the learning process for teachers as well as students. Rubrics that are designed without taking into account various variables can cause poor outcomes for students. Clear rubrics must be developed that evaluate the performance of students in an efficient and effective manner. The goal should be that the rubric should neither constrain nor diminish the learning objectives for the students. This helps to achieve long term success within a short period of time (Jackson, 2009). Rubrics should neither be narrow nor broad as they must meet the requirements in a dynamic and proficient manner. It is through the use of innovative and creative strategies that success can be attained within a short period of time.

\section{Best Practices}

Performance criteria must be clearly outlined for the success of the rubric as this leads to long term growth. The performance outcomes must be very clear so that the key features of the assignment can be outlined (Andrade \& Ying, 2005). Delivery is important part of performance criteria because the teacher must be able to assign a specific deadline for the assignment. Another important part of performance criteria is content which must be clearly outlined to the student. Organization and physicality are other components that must be present in order to assess the assignment.

The performance criteria should always be evident and measurable when the rubric is being designed. Vague learning outcomes tend to create confusion in the minds of students. Specific action verbs need to be used such as list, explain, critique, predict and others when designing a rubric for comprehension. Students should also be part of the process in creating the rubrics. This is beneficial as it can help to engage the students (Arter \& McTighe, 2001). Moreover, students are able to internalize the understanding for the criteria when they are part of the process. Students are able to make informed choices which can help them to develop insight into the required performance outcomes. The rubrics must be developed by using clear and precise goals. The performance criteria should be valid in order to achieve long term success. Performance levels are very important in the development of rubrics because they help to creating multiple rating levels. The key to success is to ensure that various rating levels can be used depending upon the objectives of the assessment.

Fewer levels are needed when collective decisions need to be made by teachers. This helps to increase the reliability of the rubrics. Multiple levels are employed when performance needs to be improved for students. Additionally, teachers might want to provide feedback to the students. The student can be given particular information about the various aspects of the performance.

Performance descriptions are very important as part of the rubric development process. This is because it helps to ensure that various performance levels can be described to the students. Adequate information must be provided to the students but it must not overwhelm them. The only goal of the description should be to develop scoring criteria for the project (Arter \& McTighe, 2001). Additionally, the rubric will play a leading role in supporting and helping the students to make informed judgments. Rubrics are important assessment tools because they help to achieve long term growth and development of students. They need to be employed in an efficient and effective manner so that they can be employed for long term success. Moreover, it is through the use of an integrated and coordinated strategy that success is achieved. Rubrics should be used in such a way that the teachers should seek to motivate and stimulate the learners to work at optimum levels. Information should be processed and developed inside the brains of students. Vocabulary is slowly built up in order to ensure that they have obtained exposure to the various language patterns and structures. They can apply outside knowledge in order to obtain an outline of the text.

Empirical studies have demonstrated that a staged approach towards rubrics can produce benefits for students (Arter \& McTighe, 2001). It helps to generate interest and passion in the students as it leads to a commitment and devotion for reading comprehension skills. Further reading helps to enhance the cognitive and intellectual capacity of students. Finally it exposes them to vast literature that can help them achieve educational objectives.

Empirical and theoretical studies have demonstrated that reading comprehension is a multifaceted and multidirectional activity. It comprises of knowledge about the external environment amalgamated with basic language structures and patterns. An interactive approach towards reading seeks to utilize a top down approach. This is because the student is interacting with the literature (Andrade \& Ying, 2005).

Based upon this interaction, the student develops a unique understanding and awareness about the entire process. Previous knowledge is also utilized in order to develop comprehension. Rubrics should be employed in such a 
manner that they help to apply an interactive approach towards learning among the students. The interactive approach helps to augment the cognitive and psychological abilities of the student. It provides a structural framework that enhances learning and reading skills. Studies recommend that a task based approach should be utilized in the educational environment.

Dividing reading into specific tasks helps to produce superior outcomes. Sentences for instance should be integrated with real situations. The entire context of the literature should focus on academic and practical knowledge. Fluency and proficiency should be encouraged in a slow and logical manner. The learners should be integrated into the classroom setting in order to achieve objectives. An interactive environment helps to increase the levels of confidence for students (Andrade \& Ying, 2005). An open atmosphere of communication and association demonstrates commitment towards developing reading skills. It helps to increase the morale of students. Further it can significantly reduce boredom associated with conventional reading materials and techniques.

Another unique and innovative approach towards the development of rubrics is the use of task oriented strategies. The teacher and student have a direct relationship in this setting. It helps to transform the process of language acquisition skills decisive and consequential. Various skills like skimming and scanning are utilized in the task oriented approach. The students should be able to deduce the central idea of the literature. Inferences should be derived by investigating signs in the text. Further each paragraph should be understood by linking it with the preceding one.

Students should have the ability to make smart presumptions about the text. The interactive function of the text should be evaluated in a systematic and logical manner. Vocabulary is enhanced through this process (Andrade \& Ying, 2005). Task oriented approach boosts the ability of students to learn new words and phrases. They develop grammatical and sentence structures in order to achieve efficiency and effectiveness. The use of optimized strategies helps to produce flawless strategies in reading. The teacher also divides students into specific groups. Each group is assigned specific tasks through this approach. Group discussion helps to identify the deductions made by each group. Student queries can be answered by the educational instructor (Andrade \& Ying, 2005). Thus the overall aim of task oriented approach is to enable the students to create their own reading skills and abilities. Such an approach helps to increase the confidence, willpower, and determination of students. It enables them to enhance reading skills in a smart and proficient manner.

Some researchers have identified the requirement of additional skills in order to develop efficient rubrics. They suggest the use of integrated skills in order to arrive at appropriate conclusions. Reading and listening should be simultaneously pursued in order to ensure that integrated skills are developed. Students should be tasked with the responsibility of identifying the main features of specified task. They should listen to their colleagues in order to enhance their reading abilities. Recitation of the literature by different students helps to create a collaborative educational environment (Andrade \& Ying, 2005).

It leads to the development of integrated skills that can be used to enhance the reading experience. Another valuable skill is to abridge the specified text. This method seeks to utilize smart and quick reading strategies. The student seeks to develop an outline inside their brains.

Knowledge acquisition skills can also be useful at this stage. Other integrated skills can include the use of diverse literature to enhance reading skills. Teachers for instance could integrate modern literature with classic literature. Such an approach helps to stimulate the mental activities of students. It helps them to gain an appreciation of the diverse nature of English language.

\section{Conclusion}

Rubrics are important aspect of assessing students reading comprehension. They should be designed through the focus on efficiency. The goals of instructors should be clear and precise. This helps to create a collaborative strategy for success. Moreover, the use of innovation and creativity can help to attain long term success for learners and teachers.

\section{References}

Andrade, H., \& Ying, D. (2005). Student perspectives on rubric-referenced assessment. Practical Assessment, (Research \& Evaluation, $10(3), 1-11$

Arter, J. \& McTighe, J. (2001). Scoring Rubrics in the Classroom. Thousand Oaks, CA: Corwin Press.

Eisner, E. (2009). The Enlightened Eye: Qualitative Inquiry and the Enhancement of Educational Practice, New York: Macmillan.

Hafner, J. C., \& Hafner, P. M. (2004). Quantitative analysis of the rubric as an assessment tool: An empirical study of student peergroup rating. International Journal of Science Education, 25(12), 1509-1528.

Heath, S. B. (2009). Ways with Words. Cambridge: Cambridge University Press. 
Jackson, P. (2009). Life in Classrooms. New York: Teachers College Press.

Stiggins, R. (2010). Student-Involved Classroom Assessment (3rd ed.). New York: Merrill. 